\title{
Propuesta de sistema de gestión para optimización de redes de transporte público
}

\author{
Jorge Erik Montiel Arguijoํㄹ Evelyn Gutiérrez León ${ }^{1}$, Chadwick Carreto Arellano², \\ Felipe Rolando Menchaca García ${ }^{1}$ \\ ${ }^{1}$ Instituto Politécnico Nacional, Escuela Superior de Ingeniería Mecánica y Eléctrica unidad \\ Zacatenco, Ciudad de México, México \\ ${ }^{2}$ Instituto Politécnico Nacional, Escuela Superior de Cómputo, Ciudad de México, México \\ jerikmontiel.isc@gmail.com, evelyngleon@hotmail.com \\ ccarretoa@ipn.mx, fmenchac@gmail.com
}

\begin{abstract}
Resumen. El presente trabajo propone el desarrollo de un sistema de monitoreo y administración inteligente de redes de transporte público utilizando una arquitectura de comunicación híbrida basada en redes de nodos inalámbricos que utilizan IPv6 y red celular (LTE, LTE-M), está destinado a describa los diferentes elementos necesarios para la recopilación de información, comunicación, procesamiento, almacenamiento y posterior despliegue de información.
\end{abstract}

Palabras clave: ciudades inteligentes, redes inalámbricas de sensores, internet de las cosas, nodos inalámbricos, arquitectura de comunicación.

\section{Proposal of a Management System for Optimization of Public Transport Networks}

\begin{abstract}
The present work proposes the development of a system of intelligent monitoring and administration of public transport networks using a hybrid communication architecture based on networks of wireless nodes using IPv6 and cellular network (LTE, LTE-M), it is intended to describe the different elements necessary for the collection of information, communication, processing, storage and subsequent deployment of information.
\end{abstract}

Keywords: smart cities, wireless sensor networks, internet of things, wireless nodes, communication architecture.

\section{Introducción}

Conforme las ciudades siguen creciendo en tamaño y población, el diseño de redes de transporte público se vuelve complicado, dada la amplia diversidad en los orígenes y destinos de los usuarios [1]. Así como la saturación de la infraestructura vehicular en las grandes ciudades a pesar de sus intentos de crecerla de acuerdo con la distribución 
de la población. Lo cual denota que, para reducir el tiempo de traslado de los usuarios es necesario implementar soluciones viales alternativas al uso del auto-móvil, aumentando la inversión en el transporte público [2 y 3] realizando un análisis integral del estado del transporte.

Esto ha generado que, actualmente a nivel global, se busquen soluciones y desarrollo orientado a transporte basadas en Internet de las cosas (IoT) que permitan, en una primera etapa, monitorear los sistemas de transporte público, con el fin de optimizar el despliegue de las unidades de transporte y con ello reducir el tiempo de traslado de los usuarios a través de las ciudades [4 a 6]. Dichas propuestas de solución son enfocadas a información recolectada de los recursos de los usuarios (datos recolectados por medio de los teléfonos inteligentes) para crear una base de datos común [3].

La Ciudad de México (CDMX) no se exime de estos problemas, dado que, su población (incluyendo los municipios conurbados del Estado de México), ha crecido rápidamente en los últimos años, lo que ha provocado que el tiempo de traslado entre los hogares y oficinas de los habitantes de la ciudad sea mayor, siendo ubicado entre las categorías de contendiente y con un nivel de aspiración importante según el índice de movilidad urbana publicado por Deloitte [7].

En el presente artículo se presenta la primera parte de una plataforma escalable que permite conectar diversas fuentes de monitoreo e información mediante una red hibrida, conformada por diferentes redes inalámbricas.

Esta plataforma está diseñada para adaptarse a las nuevas necesidades de la ciudad conforme se implemente, ofrece una forma de intercomunicación con sistemas nuevos y existentes que permita iniciar la conversión a una ciudad inteligente, atacando, primeramente, uno de los problemas más importantes en este momento: El transporte público.

La propuesta se centra en resolver en primera instancia, el reto de conocer el estado del transporte público, dividiéndolo en tres partes esenciales: En la primera parte se abordan los módulos generales de hardware y software necesarios para el correcto funcionamiento del sistema, posteriormente se describe brevemente la arquitectura de red que se utilizará y las tecnologías y protocolos necesarios para la operación de la red, finalmente en la tercera parte se describe la arquitectura general del sistema y se define la forma de comunicar la información recopilada desde la red de autobuses a un sistema de información para su posterior despliegue a los dispositivos de los usuarios.

Más adelante, se describe brevemente el análisis existente hasta el momento para el desarrollo del sistema de información y las conclusiones obtenidas.

\section{Desarrollo}

Con base en las características de los diferentes tipos de sistemas de transporte analizados (Metrobús, Trolebús y RTP) y en las diferentes necesidades de los usuarios, se propone una solución modular, escalable y mínimamente invasiva, la cual, utiliza tecnologías de nueva generación y bajo costo. Estas características permiten que sea posible la implementación de los dispositivos de monitoreo y comunicación de forma sencilla, haciendo uso de infraestructura existente. A continuación, se describen de a 
manera general los elementos que conforman el sistema a nivel de hardware y a nivel de software.

\subsection{Elementos del sistema}

Como se mencionó previamente, en la primera etapa, el sistema de gestión se comportará más como un sistema de información, en donde se recopilan datos de las diferentes unidades de transporte y las diferentes estaciones, a partir de sensores distribuidos en estaciones y unidades, así como de datos recopilados desde los dispositivos móviles de los usuarios. Es importante recalcar que este sistema busca concentrar y comunicar información de otros sistemas que se encuentran actualmente en desarrollo y no busca sustituirlos, es decir, en primera instancia y a diferencia de otros sistemas, no se recopila la totalidad de la información desde los dispositivos de los usuarios, en cambio se obtiene de la red de sensores en las unidades para su posterior despliegue al usuario mediante una plataforma móvil.

Los elementos del sistema se dividen en: Módulos de monitoreo, que incluye los módulos de autobús y módulos de estación; Aplicación web y móvil y Sistema de información.

\section{Monitoreo de autobús (A: Thread y B: Thread+LTE)}

Con el objetivo de informar del nivel de saturación de las líneas de transporte, se implementarán módulos que capaces de obtener el número de usuarios en la unidad en todo momento, de igual forma para conocer la ubicación de las unidades se instalará un módulo GPS el cual operará en conjunto con un transceptor BLE para detectar dispositivos de microlocalización "Beacons" para informar al usuario acerca de la estación en la que se encuentra por medio de una aplicación web del sistema. Como se puede ver en la fig. 1 se propone distribuir este monitoreo en dos tipos de módulos de acuerdo con el tipo de transporte en el que se implementará el sistema. Para sistemas de transporte con estaciones señalizadas, por ejemplo: RTP, se utilizarán nodos de tipo $\mathrm{B}$, en cambio, para sistemas de transporte con estaciones con estructuras fija, ejemplo $\mathrm{MB}$, se pueden utilizar nodos de tipo A o B de manera indistinta.

El diseño de los nodos es conformado de la siguiente manera:

- Unidad de control.

- Contadores de ascenso/descenso con detección de dirección.

- Módulo GPS

- Módulo de lectura de Beacons

- Módulo de comunicación Thread

- Módulo de comunicación LTE (Solo tipo B) 


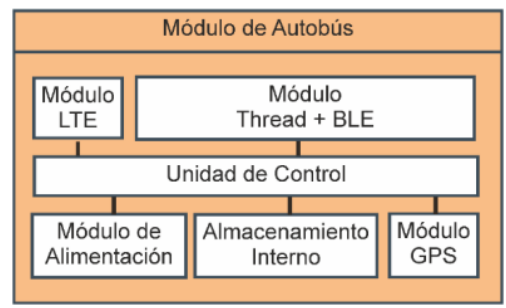

Fig. 1. Diagrama del módulo central del autobús.

Con base en la gran diversidad de tipos de unidades de transporte, se definieron diferentes módulos de monitoreo de ascenso/descenso, comunicados inalámbricamente con un módulo central mediante una red Bluetooth Mesh, otorgando una gran flexibilidad para instalar los sensores necesarios en cada unidad. La fig. 2 muestra el diagrama a bloques de los módulos de ascenso/descenso.

\begin{tabular}{|c|c|c|c|}
\hline \multicolumn{4}{|c|}{ Módulo de Monitoreo } \\
\hline $\begin{array}{c}\text { Módulo } \\
\text { BLE }\end{array}$ & \multicolumn{2}{|c|}{$\begin{array}{c}\text { Unidad de } \\
\text { Control }\end{array}$} & \multirow{2}{*}{ 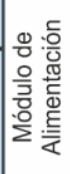 } \\
\hline $\begin{array}{l}\text { Con } \\
\text { Ultra }\end{array}$ & & $\begin{array}{l}1 \\
\text { Contador } \\
\text { Infrarrojo }\end{array}$ & \\
\hline
\end{tabular}

Fig. 2. Diagrama del módulo de monitoreo ascenso/descenso del autobús.

Los módulos de monitoreo se distribuirán en cada uno de los puntos de ascenso y descenso de la unidad, siendo los encargados de obtener la información de ascensos y descensos en cada punto de la unidad, y comunicarlos al módulo central de autobús para calcular el número de personas actual dentro de la unidad.

\section{Módulos de estación}

Como se mencionó previamente, este sistema se enfoca en distribuir los dispositivos en los autobuses, con el objetivo de garantizar versatilidad de la solución, en otras palabras, que todo sistema de transporte sea aplicable a la solución con los menores ajustes posibles. Sin embargo, es necesario para una correcta sincronización y despliegue de información al usuario, colocar módulos de identificación en las estaciones, estos módulos pueden ser completamente autónomos y con mínimo impacto en la infraestructura actual. De acuerdo con el tipo de sistema de transporte en el que se implementará la solución, se proponen dos tipos de módulos:

\section{Estaciones señalizadas}

En el caso de las rutas que sólo dispongan de estaciones señalizadas, se colocaran "Beacons" en cada estación, este dispositivo permitirá, con base en la intensidad de señal, obtener la proximidad de los autobuses respecto a la estación, esto es, si está llegando, saliendo o si está parado en la estación. Este en conjunto con el módulo GPS, se obtendrá información de la ubicación de la unidad; además este dispositivo permite 
a los usuarios que cuenten con la aplicación móvil en sus dispositivos, visualizar en qué estación se encuentran de forma automática y sin consumir de su paquete de datos o su módulo GPS, siempre y cuando sean compatibles con el protocolo a utilizar en los Beacons.

\section{Estaciones de estructura fija}

Para las estaciones con estructura fija como lo son estaciones de sistemas BRT (Metrobús, Mexibús, Algunos trolebuses, y autobuses del sistema M1) se colocará adicionalmente al Beacon un módulo de comunicación LTE, esto para brindar puntos de redundancia adicional en la red y con ello garantizar que el sistema de información siempre estará actualizado incluso sin autobuses con nodos tipo B disponibles "Fig. 3".

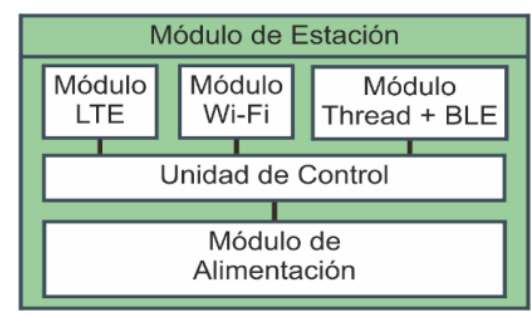

Fig. 3. Diagrama de módulo de estación con estructura fija.

Estos módulos solamente actúan como repetidores y nodos frontera de la red Thread desplegada entre las estaciones y los autobuses, la característica principal a resaltar en estos nodos es la posibilidad de adaptarlo a la tecnología de comunicación a nivel de internet que se tenga disponible en el lugar de instalación, es decir, se puede implementar la comunicación del sistema a nivel de internet a partir de un módulo, LTE, 3G, Wifi o Ethernet, entre otros.

\section{Aplicación Web}

Para el despliegue de información a los usuarios se desarrollará una aplicación web la cual permita acceder a la información del sistema desde cualquier dispositivo compatible, de igual forma se adaptará esta aplicación web para que se ejecute como una aplicación en teléfonos inteligentes, primeramente, con sistema operativo Android y posteriormente iOS.

La aplicación móvil permitiría a los usuarios del transporte visualizar el estado de las líneas incluyendo: tiempo de llegada del próximo autobús a una parada específica, nivel de saturación del autobús (cantidad de personas/capacidad especifica), diferentes rutas de transporte registradas en el sistema que paran en una misma parada, así como detectar automáticamente la parada en la que se encuentra mediante la detección del "Beacon" instalado en la parada.

En el caso de los sistemas de transporte BRTs se puede desplegar la información de los autobuses, así como su tiempo estimado de llegada en los monitores instalados dentro de las estaciones.

A los operadores y administradores de la red de transporte, la aplicación les permitirá visualizar indicadores de uso de la red incluyendo ascensos y descensos por parada, 
nivel de saturaciones de los autobuses por estación, ruta y hora, así como el tiempo entre autobuses.

Así mismo se busca que la página permita a los administradores de la red, generar indicadores de operación los cuales les permitan una mejor toma de decisiones con base en el uso de la información recopilada por el sistema en "tiempo real".

\subsection{Sistema de información}

La información recabada en los sistemas de transporte se comunicará mediante los módulos LTE con el sistema de información. Este es el encargado de procesar y almacenar toda la información de la red de forma que esta pueda ser desplegada de forma fácil y entendible mediante gráficos e indicadores de las diferentes variables del sistema.

El sistema de información se alojará en una nube privada y se gestionará mediante el uso de servicios REST. Los cuales interconectaran los diferentes nodos del sistema, así como los clientes móviles, y aplicaciones web.

\subsection{Arquitectura de red}

Para la comunicación de los diferentes puntos de monitoreo se propone una arquitectura de red híbrida conformada por una red de nodos inalámbrica la cual brinde una primera infraestructura de comunicación entre las unidades de transporte y un conjunto de enlaces LTE que permitan comunicar todos los nodos a un sistema de información en la nube el cual distribuya la información a los usuarios de la red de transporte (Fig. 4).

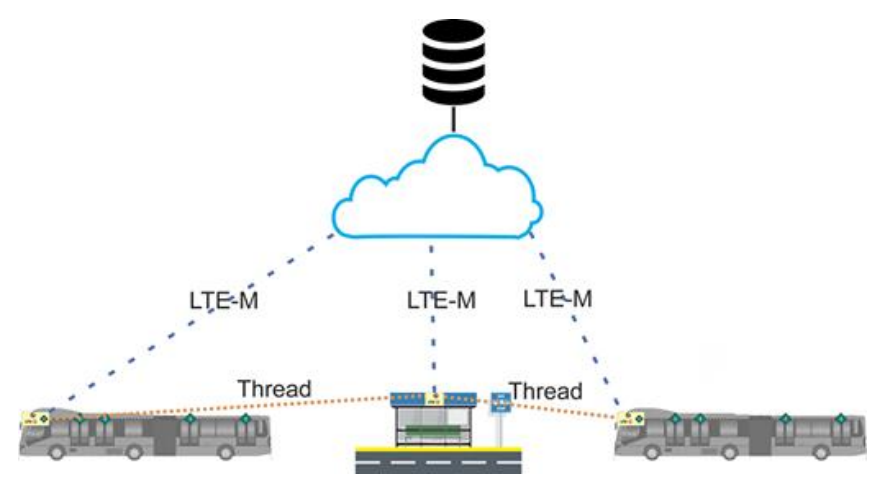

Fig. 4. Arquitectura de comunicación básica.

Dentro de las unidades de transporte es necesario colocar nodos de monitoreo en diferentes puntos de la unidad, estos puntos se comunicarán inalámbricamente mediante una red Bluetooth Mesh. La información se concentrará en el nodo principal del vehículo el cual comunicará las variables monitoreadas con los nodos cercanos utilizando la red Thread y en caso que el vehículo disponga de un modem LTE se enviará la información igualmente por este medio al sistema de información "fig. 5". 


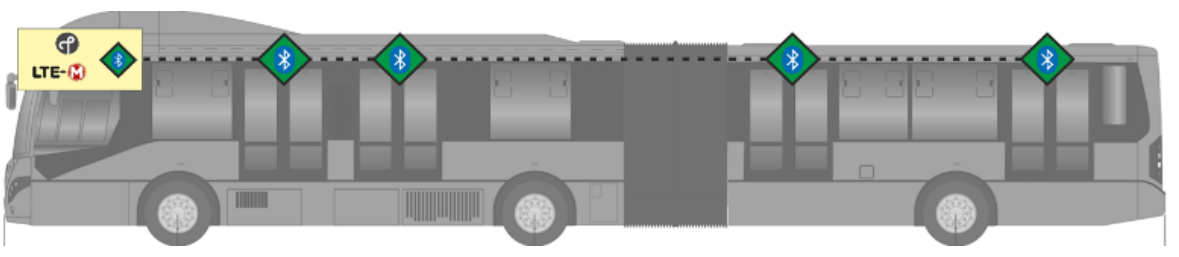

Fig. 5. Distribución de nodos en unidades de transporte.

El uso de una topología en malla permite agregar y configurar los elementos necesarios para el monitoreo de forma dinámica con base en las características del transporte específico a monitorear.

Para interconectar las diferentes unidades de transporte con las estaciones de estructura fija y posteriormente comunicarse con el sistema de información, en las estaciones se desplegarán nodos basados en Thread los cuales permitirán una comunicación directa entre estaciones y unidades de transporte optimizando la distribución de las variables monitoreadas en cada punto de forma independiente ayudando con esto a alimentar los algoritmos de aprendizaje automático que se implementarán en la red de nodos "Fig. 6", de igual forma que en las unidades de transporte se seleccionarán algunas estaciones específicas (con base en su ubicación geográfica) para incluir en su nodo un modem LTE que permita comunicar los nodos con el sistema de información.

Es importante mencionar que la red de nodos también obtendrá variables de configuración y de control del sistema de información las cuales permitirán ajustar los parámetros de los algoritmos de aprendizaje automático con base en las necesidades de los usuarios (gestores de la red de transporte).

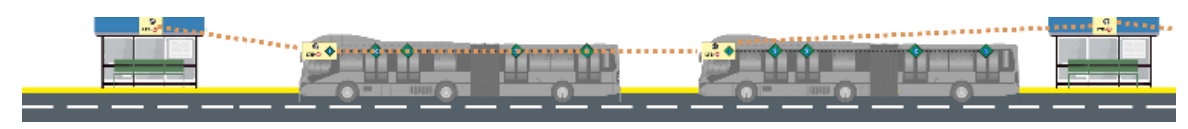

Fig. 6. Comunicación nodal entre estaciones y autobuses.

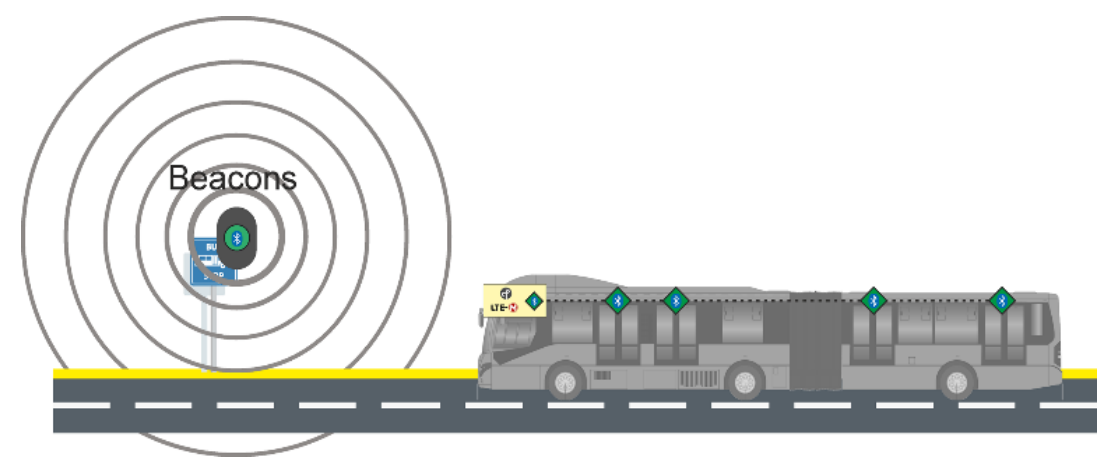

Fig. 7. Detección por proximidad en estaciones señalizadas. 
En el caso de las estaciones que no dispongan de las características necesarias para operar un nodo de comunicación, como los son aquellas conformadas solo por un letrero en una vialidad, se colocarán dispositivos de microlocalización "Beacons" los cuales permitan a las unidades de transporte conocer y posteriormente reportar la ubicación de la unidad mediante el identificador de la estación "Fig. 7"; y en el caso de los usuarios con dispositivos móviles compatibles obtener información de las próximas unidades de transporte mediante la aplicación móvil desarrollada.

Finalmente, los usuarios del sistema (pasajeros, operadores y gestores de la red de transporte) visualizarán la información recopilada del sistema mediante una aplicación web en cualquier dispositivo compatible con conexión a internet.

\subsection{Arquitectura del sistema}

Para la comunicación a nivel lógico de todos los componentes del sistema se propone una arquitectura de software modular la cual permite el monitoreo y la notificación a los módulos mediante el uso de servicios REST para el primer caso y notificaciones PUSH para el segundo.

Esta arquitectura se simplifica en gran parte en la comunicación con los nodos Thread debido a que este protocolo permite re direccionar las tramas de información de forma directa gracias a la implementación de IPv6 dentro de la comunicación de los nodos [8].

Los nodos distribuidos en los autobuses comunican la información recabada a través de la red Bluetooth Mesh desplegada dentro del autobús, posteriormente esta se comunica mediante la red Thread hasta alcanzar un nodo frontera a partir del cual se comunicará al servidor Web haciendo uso de los servicios Web.

Una vez en el servidor esta información se procesará y se almacenará en la base de datos del sistema de información y posteriormente, con base en la fuente de origen de la información se notificará a los nodos y usuarios en las estaciones pertinentes mediante los canales de notificaciones PUSH con lo cual se evitará que la aplicación Web y los nodos de la red estén realizando un "poleo" constante.

\section{Implementación}

Hasta este momento se ha llevado acabo el análisis y diseño de los módulos de monitoreo dentro del autobús los cuales manejan la cuenta de usuarios dentro de las unidades de transporte, se desarrollaron 2 prototipos de este modo, el primero utilizando el transceptor nRF24L01+ con el objetivo de comunicar los diferentes nodos instalados dentro de una unidad. Debido al modularidad del sistema se pueden modificar las características de la red de sensores inalámbrica, por ello el segundo prototipo se optó por utilizar un conjunto de sensores y transceptores diferentes, en este segundo caso, se agregó un sensor de corte infrarrojo en conjunto con los sensores de distancia infrarrojos y un XBee S2C como módulo de comunicación entre nodos. 


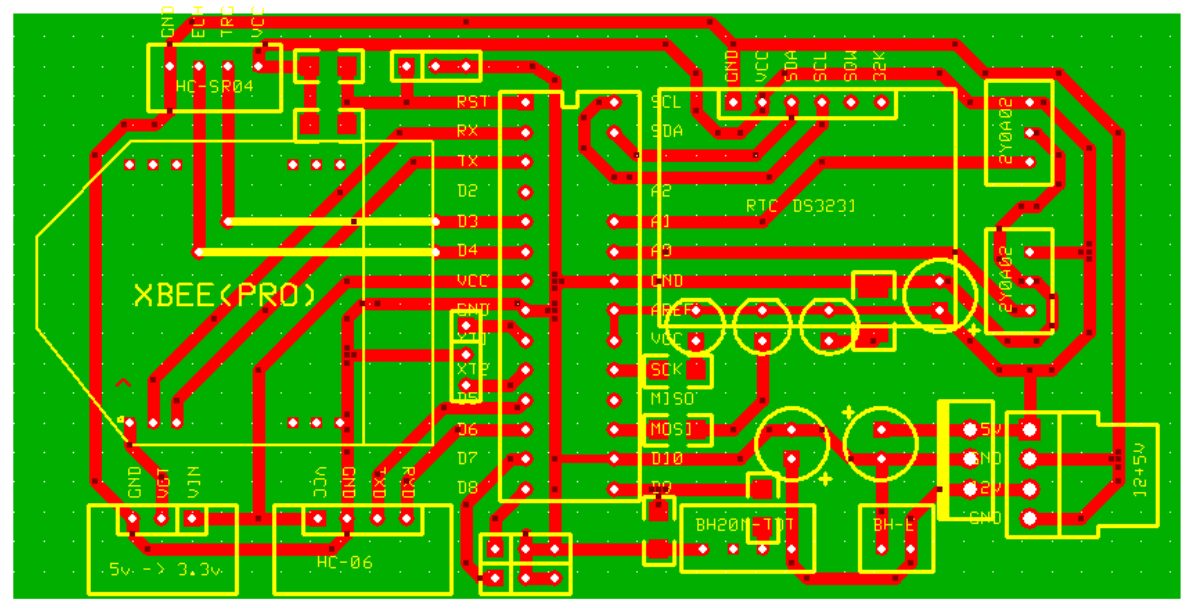

Fig. 8. Diseño de PCB de módulo de monitoreo usando XBee

Finalmente se diseñó un módulo adaptable al cual puede conectarse cualquier de los dos transceptores, así como los diferentes sensores analizados, este último diseño se realizó en EAGLE y busca ser compacto y compatible cualquiera de los transceptores probados, utilizando ZigBee a través de XBee mediante el módulo XB1 o mediante BLE a través del nRF24L01+ usando el conector JP3.

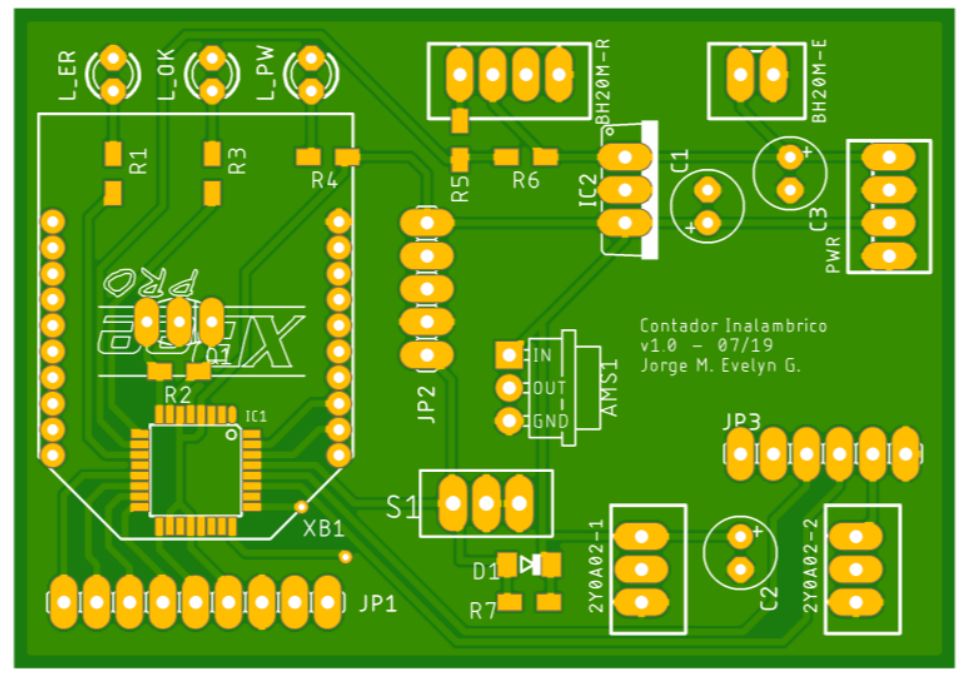

Fig. 9. Diseño de PCB de nodo de monitoreo en EAGLE

De igual forma se trabaja con tarjetas de desarrollo basadas en el nRF52840 para el despliegue de los nodos de autobús y de estación, estos módulos son los encargados de generar la red Thread en multiprotocolo con BLE para la comunicación de las diferentes unidades de transporte. 


\section{Conclusiones}

El sistema propuesto se distingue de otras implementaciones similares en la arquitectura de comunicación de los vehículos de transporte la cual le permite al nodo conocer el estado de los demás nodos además del suyo.

La propuesta tiene como principales ventajas:

- Arquitectura fácilmente escalable.

- Nodos inalámbricos que permiten alta flexibilidad en la instalación dentro de las unidades de transporte.

- Comunicación de las unidades de transporte sin necesidad de conexión a internet.

- Posibilidad de utilizar diversos tipos de unidades de transporte y diversas rutas dentro de un mismo sistema.

Como trabajo a futuro se desarrollará la red de comunicación entre autobuses y estaciones basada en el protocolo Thread, se desarrollarán los módulos de comunicación LTE-M y se desarrollará el sistema de información con las tecnologías de comunicación necesarias para poder verificar el correcto funcionamiento del sistema, igualmente se desplegarán algunos nodos en vehículos designados los cuales se monitorearán en un camino definido dentro de la ciudad esto con el fin de realizar pruebas de operación en un entorno real y verificar la funcionalidad del sistema.

Se considera en una siguiente etapa visualizar la posibilidad de integrar el pago del servicio de transporte a través de la plataforma, así como fungir como credencial de identificación para el acceso a la ruta de transporte. Esto puede ser desarrollado mediante la implementación de NFC o a través de algún código bidimensional dinámicos (QR, PDF417, etc.).

Finalmente consideramos que es importante el desarrollo de soluciones enfocadas a IoT, entre ellas a Smart Cities que permitan la integración de la diversa infraestructura existente a las nuevas tendencias en sistemas de información, de forma que se puedan optimizar los diferentes servicios que ofrecen las ciudades, reduciendo, en el caso del transporte público, los tiempos de traslado y mejorando la eficiencia en el consumo de combustible, mejorando paulatinamente la calidad de vida de los habitantes.

\section{Referencias}

1. Handte, M., Foell, S., Wagner, S., Kortuem, G., Marron, P.J.: An Internet-of-Things Enabled Connected Navigation System for Urban Bus Riders. IEEE Internet Things J. 3, 735-744 (2016). https://doi.org/10.1109/JIOT.2016.2554146

2. Instituto de investigaciones parlamentarias. Diagnóstico de movilidad en la Ciudad en México: El impacto del crecimiento vehicular (problemas, estadísticas y evaluación de políticas), 118 (2017)

3. Farkas K, Nagy, A.Z., Tomas, T., Szabo, R.: Participatory sensing based real-time public transport information service. In: 2014 IEEE Int Conf Pervasive Comput Commun Work PERCOM Work 2014, pp. 141-144 (2014). doi: 10.1109/PerComW.2014.6815181 
4. Lohokare, J., Dani, R., Sontakke, S., Adhao, R.: Scalable tracking system for public buses using IoT technologies. In: 2017 Int. Conf. Emerg. Trends Innov. ICT, ICEI 2017, pp. 104109 https://doi.org/10.1109/ETIICT.2017.7977019 (2017)

5. Raj, J.T., Sankar, J.: IoT based smart school bus monitoring and notification system. In: 5th IEEE Reg. 10 Humanit. Technol. Conf. 2017, R10-HTC 2017, pp. 89-92. https://doi.org/10.1109/R10-HTC.2017.8288913 (2018)

6. Reyes, C., Barreto, A., Bautista, V.E.: Sistema de monitoreo del LOBOBUS. 2, 998 (2015)

7. Nieblas, M.: Movilidad en la CDMX | Deloitte México. https://www2.deloitte.com/mx/ es/pages/dnoticias/ articles/movilidad-en-la-cdmx.html

8. Unwala, I., Taqvi, Z., Lu, J.: Thread: An IoT protocol. In: IEEE Green Technol. Conf, pp. 2018-April, 161-167. https://doi.org/10.1109/GreenTech.2018.00037 (2018) 